\title{
Attentional Networks in Boys With ADHD or Autism Spectrum Disorder and the Relationship With Effortful Control
}

\author{
Vicky Samyn ${ }^{1}$, Herbert Roeyers $^{1}$, Patricia Bijttebier ${ }^{2}$, and Jan R.Wiersema ${ }^{1}$ \\ ${ }^{1}$ Ghent University, Belgium \\ ${ }^{2}$ University of Leuven, Belgium
}

\section{Corresponding author}

Vicky Samyn, Ghent University, PP05, Henri Dunantlaan 2, B-9000 Ghent, Belgium Email: Vicky.Samyn@UGent.be

\section{Brief biographical statement}

Vicky Samyn, MSc, is a doctoral student in Psychology at Ghent University, Belgium.

Herbert Roeyers, $\mathrm{PhD}$, is a professor of Clinical Psychology at Ghent University, Belgium. His research interests include cognitive and social-cognitive development in ASD and ADHD. Patricia Bijttebier, PhD, is a professor of Developmental Psychopathology at the University of Leuven, Belgium.

Jan R. Wiersema, PhD, is a professor of Clinical Psychology at Ghent University, Belgium. 


\begin{abstract}
Objective: The present study investigated differences in attentional networks in typically developing (TD) boys and boys with ADHD or autism spectrum disorder (ASD). Additionally, we investigated the relationship between networks and the relationship with Effortful Control (EC). Method: An Attention Network Test was used to assess alerting, orienting, and executive attention in 25 TD boys, 25 boys with ADHD, and 25 boys with ASD. Results: In the absence of warning signals, boys with ADHD performed worse than other children. In all groups, the orienting and executive control networks and the alerting and orienting networks interacted. Executive attention and EC were unrelated. Conclusion: Results provided evidence of impaired tonic alertness in ADHD and support the idea of functional integration of attentional networks. Finally, findings suggest that the link between EC reports and indices of neural systems involved in the effortful regulation of behavior may not be as unambiguous as previously thought.
\end{abstract}


Attention serves as a basic set of mechanisms that underlie our awareness of the world and the voluntary regulation of our thoughts and feelings (Posner \& Rothbart, 2007). Posner and Petersen (1990) distinguish three attentional networks, each having a different function and corresponding to separable brain regions and neurochemical circuits. The alerting network involves achieving and maintaining an alert state. Although not all studies explicitly make a distinction between different aspects of alerting, two types of alertness can be distinguished. Tonic alertness or vigilance which refers to an internal alertness or to sustained activation over a period of time, and phasic alertness which refers to the nonspecific activation that takes place when a warning signal is presented (Callejas, Lupiàñez, Funes, \& Tudela, 2005). The alerting system has been linked to the frontal and parietal regions of the right hemisphere (Fan, McCandliss, Sommer, Raze, \& Posner, 2002) and to the brain's norepinephrine system, with tonic levels of alertness driven by noradrenergic input from the locus coeruleus (e.g., Posner \& Peterson, 1990). The orienting network involves the selective allocation of attention to a potentially relevant area of the visual field and has been associated with areas of the parietal and frontal lobes (Fan et al., 2002) and with the cholinergic system (e.g., Greenwood, Fossella, \& Parasuraman, 2005). Finally, the executive attention or conflict network involves the monitoring and resolving of conflict among thoughts, feelings, and responses and has been linked to the anterior cingulate cortex (ACC) and the lateral prefrontal cortex (LPFC; e.g., Posner \& Fan, 2004) and to the dopaminergec system (e.g., Bush, Luu, \& Posner, 2000). Because of the fact that executive attention provides a basis for the ability to regulate behavior, it is believed to be related to Effortful Control (EC), the self-regulation component of temperament (Rothbart \& Bates, 2006). Posner and Rothbart (1994) have argued that, although EC is a heterogeneous construct involving multiple systems, the executive attention network is at the core of the capacity to effortfully regulate behavior. Moreover, they have reinforced this theoretical link by including this network in the broader definition of EC: "the 
efficiency of executive attention, including the ability to inhibit a dominant response and/or to activate a subdominant response, to plan, and to detect errors" (Rothbart \& Bates, 2006, p. 129). The theoretical link between EC and the executive attention network has brought forth several studies that investigated the relationship between EC reports and performance on executive attention tasks (e.g., Ellis, Rothbart, \& Posner, 2004; Gerardi-Coulton, 2000; Simonds, Kieras, Rueda, \& Rothbart, 2007). Overall, there is some evidence for a relationship between EC and executive attention, but especially when parent-reports are considered. In addition, results seem to depend on the type of neuropsychological measure that is used to tap executive attention. Given the fact that findings on the relationship between EC and executive attention are not completely univocal, further research may be warranted to help clarify the exact relationship between both constructs.

The Attention Network Test (ANT) was developed by Fan and colleagues (2002) to assess the different attentional networks (and their interrelations) within a single task. In specific, the efficiency of alerting, orienting and executive attention is measured by evaluating the impact of an alerting cue, spatial cues and flankers on reaction time. Originally, the attentional networks were assumed to be related to independent aspects of attention (Fan et al., 2009). In the original report of their work, Fan and colleagues (2002) did find support for independence between networks. However, they also reported an interaction between cue condition and flanker type, suggesting that the attentional networks may not be completely independent. Callejas and colleagues (e.g., Callejas et al., 2005) have argued that these interaction effects can not be interpreted since in the original ANT, alerting and orienting are measured with different levels of the same variable (i.e., double cue and spatial cues, respectively). In order to make it possible to measure the networks independently and test the influence of each network on the other networks, Callejas, Lupiáñez, and Tudela (2004) developed a modified ANT, in which the alerting signal was a short duration high frequency 
tone (instead of the double cue). Since then, several studies have confirmed that in healthy controls, the attentional networks are not as independent as originally thought and have supported the idea of functional integration of and interaction between the different networks (e.g., Callejas et al., 2004; Fan et al., 2009).

The conceptualization of attention as an organ system with three specialized neurofunctional networks (Posner \& Fan, 2004) can possibly shed a light on differences in attentional modulation between typically developing (TD) children and children with atypical attentional processes (Posner \& Petersen, 1990). One disorder that is characterized by deficient attentional functions is attention deficit/hyperactivity disorder (ADHD; Konrad, Nuefang, Hanisch, Fink, \& Herpertz-Dahlmann, 2006). Berger and Posner (2000) have attempted to conceptualize three recent theoretical accounts on ADHD as pathologies of attentional networks. They describe the theory of Swanson and colleagues (Swanson et al., 2000) as a combination of executive attention and alerting deficits, whereas the functions included in Barkley's theoretical account (1998) are conceptualized as part of the executive attention network. Finally, they argue that the theory of Sergeant and colleagues (1999) that emphasizes on energetic factors as the most critical deficit in ADHD, could also be seen as part of the executive control network. The fact that this conceptualization puts forward executive attention and alerting as the networks of interest in ADHD, is in line with neurochemical studies identifying dopamine (involved in executive attention network) and norepinephrine (involved in the alerting network) as major players in the pathophysiology of ADHD (e.g., Sengupta et al., 2002). Several studies have investigated attentional networks in children with ADHD and studies using the ANT are largely consistent in that they showed no orienting deficit in comparison with TD peers (e.g., Adólfsdóttir, Sorensen, \& Lundervold, 2008; Booth, Carlson, \& Tucker, 2007; Johnson et al., 2008; Konrad et al., 2006). Findings on alerting and executive attention are less consistent. Some studies showed differences in the efficiency of 
executive attention (i.e., a larger interference effect in the ADHD group; e.g., Konrad et al., 2006; Mullane, Corkum, Klein, McLaughlin, \& Lawrence, 2011), and/or in the efficiency of alerting (e.g., Casagrande et al., 2012; Johnson et al., 2008; Mullane et al., 2011) whereas others showed no differences in comparison to TD peers (e.g., Adólfsdóttir et al., 2008; Booth et al., 2007).

Another disorder that is known to show atypical attentional processes is autism spectrum disorder (ASD; e.g., Allen \& Courchesne, 2001). Although attention deficits are not considered to be a core characteristic of ASD, attention to socially-salient stimuli may be of particular interest to understanding ASD impairments. Several authors have proposed that attentional impairments may be related to the manifestation of problems that characterize ASD (e.g., understanding and reacting to everyday situations; e.g. Courchesne, 1995). Because attentional processing is a key component in the execution of complex cognitive actions, an early-occurring attention deficit may interfere with the development of higher-order cognitive and social skills (e.g., executive functioning, joint attention). In turn, this might result in behavior problems that are typical of ASD such as difficulty coping with change, a tendency to focus on details in the environment, and a tendency to engage in repetitive and stereotypical behavior (Swettenham et al., 1998). Although previous research indicates that individuals with ASD show impairments in several attention processes (for an extensive review, see Keehn, Lincoln, Müller, \& Townsend, 2010), they seem to have a particular problem with spatial attention function or orienting (e.g., Haist, Adamo, Westerfield, Courchesne, \& Townsend, 2005). To the best of our knowledge, only one study thus far investigated all three networks within a single sample of children with ASD. Keehn and colleagues (2010) used the ANT to compare the efficiency of attentional networks in children and adolescents with ASD and TD controls. Results indicated that only the orienting network was impaired in children with ASD. 
We are not aware of any study directly comparing children with ADHD and children with ASD in terms of the efficiency of attentional networks, despite the fact that the comparison between both groups could provide us with valuable information. Several studies have shown that children with ADHD and children with ASD share many symptoms, including attention deficit and overactivity (e.g., Mayes, Calhoun, Mayes, \& Molitoris, 2012) which may complicate differentiation between the two disorders and may create a falsely inflated rate of comorbid ADHD in ASD, up to 78\% (for a review, see Gargaro, Rinehart, Bradshaw, Tonge, \& Sheppard, 2011). Examining differences in the efficiency of alerting, orienting, and executive attention may identify cognitive markers that can help distinguish between both disorders and identify false cases of comorbidity. In addition to the fact that there are no studies directly comparing children with ADHD and children with ASD on the efficiency of attentional networks, only a limited number of studies investigating these networks in ADHD or ASD, have focused on the interrelationship between the networks. Only in the study of Keehn and colleagues (2010) the relationship between alerting, orienting, and executive attention was explicitly evaluated in a TD and clinical group separately. Using correlational analyses, they found the networks to be unrelated in TD children, whereas in the ASD group, a positive relationship between alerting and executive attention emerged. However, correlational analyses are only one way to evaluate the independence of the networks. A second way to investigate potential group differences in the interrelationship between the networks (e.g., Fan et al., 2002) is by looking at potential interaction effects between group and the experimental factors that influence alerting (the presence of a warning signal), orienting (the presence of a spatial cue), and executive attention (congruent versus incongruent flankers). In their paper, Keehn and colleagues (2010) reported no significant three-way interactions involving group, suggesting that interactions between the networks were very similar in TD children and in children with ASD. If we look at ANT studies using comparable analyses to investigate 
attentional networks in children with ADHD, findings are less clear with some studies showing similar interactions between groups (e.g., Konrad et al., 2006) and others suggesting that the interaction between networks may differ between children with ADHD and TD children (e.g., Casagrande et al., 2012). We are not aware of any study investigating potential differences in the relationship among networks between both clinical groups.

The first aim of the present study is to investigate the efficiency of attentional networks in TD children and in children with ADHD or ASD. Based on the above mentioned theoretical accounts and based on previous ANT research we expect children with ASD to show lower orienting scores and children with ADHD to show larger alerting and/or executive attention scores as compared to TD children. Given the lack of research comparing children with ADHD and ASD on attentional networks, it is more difficult to put forward specific hypotheses as to potential differences between both clinical groups. Based on previous findings comparing both clinical groups to TD peers, we expect children with ASD to show more difficulties with orienting as compared to children with ADHD and we expect the latter group to show more problems with alerting and/or executive attention than their peers with ASD. The second aim of our study is to investigate the relationship between attentional networks and whether or not the networks interact differently in these three groups. Although the three networks correspond to separable brain regions, we expect to find significant interactions between the networks based on the idea that brain areas must work together to perform even the simplest task (e.g., Posner, Sheese, Odludas, \& Tang, 2006). Also, we expect these interactions to be present in all groups. A third, additional aim of our study is to examine the relationship between EC reports and the efficiency of executive attention. Given the strong theoretical link between both constructs, we expect lower executive attention or interference scores to be significantly related to higher levels of EC.

\section{Method}




\section{Participants}

Seventy-five boys aged 10-15 years with an estimated full scale IQ (FSIQ) of 80 or higher participated. TD boys $(n=25$; age: $M=12.94, S D=1.45$; estimated FSIQ: $M=104.92$, $S D=10.23)$ were recruited through advertisements. Boys with ASD diagnosis $(n=25$; age: $M$ $=12.75, S D=1.49$; estimated FSIQ: $M=101.24, S D=11.45)$ or ADHD diagnosis $(n=25$; age: $M=13.16, S D=1.61$; estimated FSIQ: $M=107.92, S D=12.11)$ were recruited through special school services. Groups did not differ from each other in terms of age $(F(2,72)=.47$, $p=.629)$ or estimated FSIQ $(F(2,72)=2.20, p=.119)$.

All children with ASD or ADHD were previously diagnosed by a multidisciplinary team using established criteria, as specified in DSM-IV-TR (APA, 2000). Diagnosis of ADHD was verified using the disruptive behavior module of the Diagnostic Interview Schedule for Children for DSM-IV (DISC-IV; Shaffer, Fisher, Lucas, Dulcan, \& Schwab-Stone, 2000). The DISC-IV was also used for establishing the presence of comorbid Oppositional-Defiant Disorder (ODD) and Conduct Disorder (CD). The ADHD group included 12 primarily Inattentive type, 1 primarily Hyperactive/Impulsive type and 12 Combined subtype. Five boys also met criteria for ODD, and 1 boy met criteria for both ODD and CD. 21 of the boys were taking medication for ADHD symptoms, but stopped taking medication at least 24 hours previous to the testing. Diagnosis of ASD was confirmed by the SRS. Forty percent of the boys in the ASD group had an SRS Total T-score between 60 and 75, indicating the presence of mild ASD or high functioning autism. Sixty percent of the boys had T-score of over 75, indicating the presence of severe autism. In the ASD group no children were taking medication.

Ratings on the Disruptive Behavior Disorder Rating Scale (DBD; Pelham, Gnagy, Greenslade, \& Milich, 1992) and the Social Responsiveness Scale (SRS; Constantino \& Gruber, 2005) were used to screen TD boys for ADHD and ASD symptoms. Boys with a DBD 
normative score of 15 or higher on the Inattention and/or Hyperactivity/Impulsivity subscale or 16 or higher on the ODD and/or CD subscale, and boys with an SRS Total T-score of 60 or higher were excluded from the study. Boys with ASD were screened for ADHD symptoms, using ratings on the DBD. As an exclusion criterion, we used a clinically significant score for ADHD Inattentive type or ADHD Hyperactive/Impulsive type as reported by parents and teachers. However, none of the children in the ASD group met this criterion. Boys with ADHD were screened for ASD symptoms, using ratings on the SRS. As an exclusion criterion, we used an SRS Total T-score of 60 or higher. Originally, 27 boys with ADHD participated in our study, however 2 boys met this criterion and were excluded from the study.

\section{Measures}

Attention network test (ANT). We used a modified ANT, similar to the one used by Callejas and colleagues (2005). Participants had to evaluate whether the middle arrow of 5 horizontally arranged arrows is pointing left or right by pressing one of two possible keys in the keyboard. The efficiency of attentional networks was assessed by measuring the impact of an alerting cue, spatial cues and flankers on reaction time. Figure 1 illustrates the sequence of events for a single trial. First, a fixation cross of variable duration (400-1600 ms) was presented, followed by the $50 \mathrm{~ms}$ alerting signal in half of the trials. After $400 \mathrm{~ms}$ stimulus onset asynchrony (SOA), an orienting cue was presented for $50 \mathrm{~ms}$ above or below the fixation cross on $2 / 3$ of the trials. After another $150 \mathrm{~ms} \mathrm{SOA}$, target and flankers were presented on the same or opposite locations of the orienting cue and remained on the screen for $3000 \mathrm{~ms}$ or until a response was given. After the response, the fixation cross was kept on screen for a variable duration dependent on the initial duration of the fixation cross and the RT of the participant, so that each trial lasted $5350 \mathrm{~ms}$.

The experiment had a 2 (auditory signal) x 3 (visual cue) x 2 (congruency) factorial design. The auditory signal had two levels: presence or absence of a $2000 \mathrm{~Hz}$ and $50 \mathrm{~ms}$ 
sound. The visual cue had three levels: no cue (no orienting cue was presented), valid cue (orienting cue presented at same location as the following target) and invalid cue trials (orienting cue presented at the location opposite the target). Congruency had two levels: congruent (target and flankers pointed in the same direction) and incongruent trials (flankers pointed in the opposite direction to the target). The neutral condition of the congruency variable was not included since Callejas and colleagues (2005) found no differences in performance between congruent and neutral trials and adding this condition would needlessly prolong the task. The number of trials per level of each variable was kept constant. The practice block had 10 trials and was followed by three blocks of 48 test trials. Within each block, trials were presented randomly. Alerting scores were calculated by subtracting mean $\mathrm{RT}$ in the tone condition from the no tone condition (collapsed across cue and flanker conditions). The underlying idea is that the presentation of a warning tone will alert participants to an upcoming target, and because such a warning signal is not presented in the no tone condition, the RT difference between these two conditions should provide a measure of the efficiency of alerting. Orienting scores were calculated by subtracting mean RT in the valid cue condition from the invalid condition (collapsed across flanker and across tone conditions). The logic is that presenting a valid cue will inform the participant of the exact location of the target, whereas the invalid cue has nu informative value for the location of the target. Therefore, when presented with a valid cue, participants will be able to orient their attention toward the target location before its appearance. As a result, the RT difference between the valid and invalid cue conditions should provide a measure of the efficiency of orienting. Executive attention scores were calculated by subtracting mean RT in the congruent flanker condition from the incongruent flanker condition (collapsed across tone and cue conditions). In the congruent condition, the target will be accompanied by congruent flankers, pointing in the same direction as the target and therefore eliciting the same response. In the 
incongruent condition, flankers will provide conflicting information about the target response and conflict resolution will be required for participants in order to be able to respond to the target. Therefore, the flanker interference effect (i.e., RT difference between congruent and incongruent conditions) should provide a measure of executive attention. One could argue that including incongruent flanker trials in the alerting and orienting indices or tone trials in the orienting index, might have influenced our findings. Therefore analyses were repeated using alerting and orienting indices without incongruent flanker trials and orienting indices without tone trials. All results remained the same.

[Insert Figure 1 here]

Children's Effortful Control. The Effortful Control Scale (ECS), The Attentional Control Scale (ACS) and the Early Adolescent Temperament Questionnaire-Revised (EATQR) were used to tap EC. The ECS (Lonigan \& Phillips, 2001) is a self-report questionnaire measuring behavioral and attentional aspects of EC. It consists of 24 items to be rated on a 5point Likert scale. It yields a total score $(\alpha=.87)$ and two subscale scores, namely Persistence/Low distractibility (12 items; e.g., "I have a hard time concentrating on my work because I'm always thinking about other things" and "I have difficulty completing assignments on time"; $\alpha=.84$ ) and Impulsivity (12 items; e.g., "I can easily stop an activity when told to do so"; $\alpha=.69$ ). Lower scores on the Impulsivity subscale indicate higher levels of impulsivity. The ECS shows acceptable psychometric properties (e.g., Verstraeten, Vasey, Claes, \& Bijttebier, 2010).

The ACS (Derryberry \& Reed, 2002) measures the ability to focus and shift attention and consists of 20 self-report items to be rated on a 4-point Likert scale. It yields a total score $(\alpha=.81)$ and two subscale scores, namely Attention Focusing (9 items; e.g., "My concentration is good even if there is music in the room around me"; $\alpha=.70$ ) and Attention Shifting (11 
items; e.g., "I can quickly switch from one task to another"; $\alpha=.69$ ). The ACS shows acceptable psychometric properties (e.g., Verstraeten et al., 2010).

The EATQ-R (Ellis \& Rothbart, 2001) self-report consists of 65 items, the parent-report version consists of 62 items. Items are grouped into 12 clusters and 4 higher-order scales (Positive Reactivity, Negative Affectivity, Affiliativeness and Effortful Control) and have to be rated on a 5-point Likert scale. For the purpose of this study, only the EC scale ( $\alpha=.90$ for the EATQ-R-p, $\alpha=.84$ for the EATQ-R-s), consisting of the item clusters Inhibitory control (e.g., "When someone tells me to stop doing something, it is easy for me to stop"), Attentional control (e.g., "I pay close attention when someone tells me how to do something") and Activation control (e.g., "I put off working on projects until right before they're due"), was included. The EATQ-R shows acceptable psychometric properties (e.g., Muris \& Meesters, 2009).

Reports of children's behavior. The DBD (Pelham et al., 1992) contains 42 items that are scored on a 4-point scale. Parents as well as the child's teacher completed the DBD. For the purpose of the present study, analyses focused on the ADHD inattentive subscale $(\alpha=.95$ for parent-report version, $\alpha=.93$ for teacher-report version) and the ADHD hyperactive/impulsive subscale $(\alpha=.92$ for parent-report version, $\alpha=.93$ for teacher-report version).

The SRS (Constantino \& Gruber, 2005) was completed by parents. It consists of 65 items to be rated on a 4-point scale and covers the various dimensions of interpersonal behavior, communication, and repetitive/stereotypic behavior that are characteristic of ASD. For the purpose of this study, analyses focused on the SRS Total score $(\alpha=.95)$. Agreement between the ADI-R and the SRS completed by parents is $89.7 \%$ in a sample of adolescents with suspected ASD (Murray, Mayes, \& Smith, 2011).

\section{Procedure}


Once parents were informed about the aims of the study and written consents were obtained, we first asked parents, children and teachers to complete the questionnaires. Second, parents and children visited the laboratory where the ANT was administered and intellectual functioning of the children was estimated based on four subtests (Vocabulary, Similarities, Picture Arrangement and Block Design) of the Wechsler Intelligence Scale for Children III (WISC-III; Kort et al., 2002). The estimated FSIQ correlates strongly with FSIQ (Grégoire, 2000).

\section{Results}

\section{ANT main analysis - Incorrect responses}

Mean error rates were entered into a mixed-model repeated measures ANOVA with group (TD, ASD, ADHD) as between-subject factor and tone (tone, no tone), cue (no, valid, invalid) and flanker (congruent, incongruent) as within-subject factors.

There was a main effect of flanker, $F(1,72)=48.22, p=.000, \eta^{2}=.40$, reflecting more errors in the presence of incongruent flankers. There was no significant difference for the mean error rate between the TD (2.2\%), the ASD (3.6\%) and the ADHD (2.2\%) group, nor were there main effects of tone or cue.

There was an interaction effect of tone and group, $F(2,144)=4.51, p=.014, \eta^{2}=.11$. As illustrated by Figure 2, there was no effect of tone in the ADHD or ASD group, boys made a similar amount of errors whether a warning tone was presented or not. In the TD group, boys made more errors in the presence of a warning tone $\left(F(1,24)=5.53, p=.027, \eta^{2}=.19\right)$.

[Insert Figure 2 here]

There was an interaction effect of cue and flanker, $F(2,144)=5.29, p=.006, \eta^{2}=.07$. As illustrated by Figure 2, there was no effect of cue type in the presence of congruent flankers. In the presence of incongruent flankers, participants made significantly fewer errors 
in the valid cue condition than in the invalid cue condition $\left(F(1,72)=11.78, p=.001, \eta^{2}=\right.$ $.14)$.

\section{ANT main analysis - Reaction time}

Mean reaction times $(\mathrm{RT})$ for correct trials with $\mathrm{RT} \geq 150 \mathrm{~ms}(0.07 \%$ of the trials were excluded based on this criterion) were entered into a mixed-model repeated measures ANOVA with group (TD, ASD, ADHD) as between-subject factor and tone (tone, no tone), cue (no, valid, invalid) and flanker (congruent, incongruent) as within-subject factors.

We found main effects for group $\left(F(1,72)=3.20, p=.047, \eta^{2}=.08\right)$, tone $(F(1,72)=$ 72.42, $\left.p=.000, \eta^{2}=.50\right)$ and flanker $\left(F(1,72)=386.07, p=.000, \eta^{2}=.84\right)$. Boys with ADHD reacted slower to the target as compared to their TD peers. All groups responded faster to the target in the valid cue condition as compared to other cue conditions and in the no cue condition as compared to the invalid cue condition and all groups responded slower to the target if it was accompanied by incongruent flankers.

We also found an interaction effect of tone and group $\left(F(2,144)=6.09, p=.004, \eta^{2}=\right.$ $.15)$, tone and cue $\left(F(2,144)=8.03, p=.000, \eta^{2}=.10\right)$, and cue and flanker $(F(2,144)=6.76$, $\left.p=.002, \eta^{2}=.09\right)$. As illustrated by Figure 3, the facilitating effect of a warning tone was larger in the ADHD group in comparison to the TD group. For all groups, the facilitating effect of the warning tone was larger in the no cue condition than in the invalid cue condition and the facilitating effect of the congruent flankers was larger in the invalid cue condition as compared to the valid cue condition. There was no significant interaction effect of tone and flanker $(F(2$, 144) $\left.=1.15, p=.288, \eta^{2}=.01\right)$.

[Insert Figure 3 here]

\section{Group comparisons on attentional network scores}

We compared groups on attentional network scores by means of ANOVAs and Bonferroni post hoc analyses, using an overall alpha level of .05. Means, standard deviations and $F$ values are 
shown in Table 1. Significant group differences were found for the efficiency of alerting. Boys with ADHD show a larger alerting effect than TD boys and boys with ASD. Additional analyses evaluating the tone and no tone condition separately, showed that boys with ADHD responded significantly slower than TD children in the absence of a warning tone $(F(2,72)=$ $3.988, p=.023$ ) but that they performed at a similar level as the other children when a warning tone was presented $(F(2,72)=1,642, p=.201)$. Groups did not differ in terms of orienting and executive attention. Results remained the same when controlling for FSIQ. Analyses were repeated using median RT to enable the comparison with results of studies using median RT based network scores, all results remained the same. Additional analyses excluding children with ODD and/or CD from the ADHD group yielded similar results in that the three groups did not differ from each other in terms of orienting and executive attention and that children with ADHD showed a significantly larger alerting effect as compared to TD children.

Although ANT studies traditionally analyse difference scores, this method has been criticized (Keehn et al., 2010). Comparable analyses using mean RTs in ANOVAs for each network to examine interactions between group and tone, cue and flanker condition (as reported under the heading 'ANT main analysis - Reaction time'), support the findings of the difference score analyses in that there were no group differences in terms of orienting and executive attention and in that boys with ADHD showed an alerting deficit in comparison with TD boys.

[Insert Table 1 here]

\section{The relationship between network scores, FSIQ and age}

Bivariate correlations between attentional network scores, FSIQ and age were computed. We found no significant relationship between age and any of the network scores or between FSIQ and alerting or orienting scores. For the total group, a significant negative relationship between executive attention and FSIQ $(r=-.45, p=.000)$ emerged. When looking at each group 
separately, higher FSIQ was associated with a smaller interference effect in TD boys $(r=-.53$, $p=.006)$ and boys with ADHD $(r=-.71, p=.000)$, but not in boys with ASD.

\section{The interrelationship between network scores}

Bivariate correlations between alerting, orienting and executive attention scores were computed for each group separately. We found no significant relationship between the three networks in TD boys, boys with ADHD and boys with ASD.

\section{The relationship between executive attention and $\mathrm{EC}$}

Bivariate correlations between executive attention scores and EC (sub)scales were computed. For the total group, we found no significant relationship between executive attention and any of the EC (sub)scales, with the exception of the attention shifting subscale of the ACS ( $r=-$ $.25, p=.033)$. When controlling for differences in FSIQ, this relationship lost significance. When looking at the groups separately, results were identical showing no significant relationship between executive attention and EC, also after controlling for FSIQ.

\section{Discussion}

This study had three aims. The first goal was to investigate differences in alerting, orienting and executive attention between TD boys, boys with ADHD and boys with ASD. Groups did not differ in terms of orienting and executive attention. However, boys with ADHD did demonstrate a larger alerting effect than their TD peers. The alerting property of the warning signal was effective for all children, but its beneficial effect was significantly larger in boys with ADHD than in TD boys and it even enabled the ADHD group to perform at a similar level as the other groups. Overall, our results show that boys with ADHD appear to have difficulties with maintaining an alert state, but only in the absence of a warning signal. This finding is in line with previous studies (e.g., Johnson et al., 2008) and indicates that boys with ADHD show problems with tonic, but not with phasic alertness. Tonic aspects of alertness are believed to be modulated by noradrenaline and alertness problems might occur due to deficient fronto- 
parietal control over the locus coeruleus (for a review, see Halperin \& Schulz, 2006). In all, results support the growing body of evidence for an alerting deficit in children with ADHD (e.g., Casagrande et al., 2012; Mullane et al., 2011). They are also consistent with the hypothesis that increasing alertness may buffer the attention deficit in children with ADHD (e.g., Booth et al., 2007; Casagrande et al., 2012). Contrary to our expectations and to a previous finding (Keehn et al., 2010), children with ASD did not show deficient orienting as compared to TD peers. One possible explanation for these inconsistent findings may lie in sample characteristics. Our participants were boys between 10 and 15 years of age, while Keehn and colleagues (2010) included boys as well as girls from a broader age range (i.e., 8 to 19 years of age). However, this explanation seems unlikely given previous findings showing that the orienting network remains stable during brain development (Rueda et al., 2004) and, at a behavioral level, is not influenced by gender (Neuhaus et al., 2009). Another possible explanation may be methodological differences in the administered ANT. We used an auditory alerting signal whereas Keehn and colleagues (2010) used a visual cue. Although alerting signals are not explicitly taken into consideration when evaluating the orienting network, tone trials were included when calculating orienting scores and one might argue that this may have influenced these scores. However, analyses excluding tone trials yielded the same results, indicating that differences in modality of the alerting signal do not account for the inconsistent findings. An alternative explanation is that the calculation method of network scores influenced the results. We calculated orienting scores by subtracting mean RT in the valid cue condition from the invalid cue condition, whereas Keehn and colleagues (2010) subtracted median RT in the spatial cue condition (only valid cues) from the center cue condition. It was not possible to test this hypothesis since, in accordance with Callejas and colleagues (2005), we did not include center cue trials in our ANT. Therefore, this remains an open question for future research. Finally, one could argue that the fact that we did not find an orienting deficit 
in ASD as compared to TD children, may be due to the nature of the stimuli that were presented. In the ANT only nonsocial stimuli are used, whereas several studies have suggested that children with ASD may have a specific deficit in attending to socially-salient stimuli (e.g., Swettenham et al., 1998). Given these findings, it is possible that attention tasks using social stimuli may be more efficient in identifying attention deficits in ASD. Future research using an ANT with social stimuli will have to confirm whether or not these tasks are better suited to investigate alerting, orienting, and/or executive attention deficits in ASD. In addition, using the ANT of Callejas and colleagues (2005), we were unable to identify cognitive markers that can differentiate between boys with ADHD and boys with ASD. Future research will have to show whether or not an ANT with socially-salient stimuli is more efficient to this end.

A second aim was to investigate the relationship between attentional networks and whether or not the networks interact differently in TD boys, boys with ADHD, and boys with ASD. A first way to assess the interrelationship between the alerting, orienting, and executive attention networks is looking at the correlations between the networks (e.g., Fan et al., 2002). We found no significant relationship between network scores in TD boys, boys with ADHD and boys with ASD, suggesting that these attentional networks function independently. However, a second way to evaluate the relationship between the networks, involves examining interaction effects between the experimental factors that influence alerting, orienting, and executive attention by means of ANOVA (e.g., Fan et al., 2002). We found a significant interaction between tone and cue and between cue and flanker. In specific, for all groups the alerting effect was smaller in the invalid cue condition than in the no cue condition. A possible interpretation of this finding is that the facilitating effect of a warning signal is partially diminished when it is followed by an invalid cue. An invalid cue will direct attention away from the place where the target will appear and consequently, participants will need extra time to respond because they will have to redirect their attention to the target. With regards to the 
interaction between cue and flanker, we found that the congruency effect was significantly larger for trials with invalid cues than for validly cued trials. A possible interpretation of this interaction is that the adverse effect of the presence of incongruent flankers on reaction time adds to the adverse effect of directing attention in the wrong way by presenting an invalid cue. Regardless of the interpretation, our results suggest that the orienting and executive control network influence each other and that the same is the case for the alerting and orienting network. Overall, our findings are in line with previous research in that they support the idea that the networks do not operate independently in all situations and that there seems to be a functional integration between the different networks (e.g., Callejas et al., 2004; Fan et al., 2002). Our results show identical results for all groups, suggesting that the attentional networks interact in a very similar way in TD boys, boys with ADHD and boys with ASD.

A final aim was to investigate the relationship between executive attention and EC. Despite the strong theoretical link between both constructs, we found no significant relationship between EC reports and executive attention scores, regardless of the EC measures that were considered. Results remained the same when looking at the three groups separately and after controlling for FSIQ. The lack of a significant relationship between self-reports on EC and executive attention is in line with previous ANT studies (e.g., Ellis et al., 2004; Simonds et al., 2007) and is not particularly surprising, given the fact that behavioral selfreport data and neurocognitive performance data generally do not correlate very well in children. However, the fact that parent-reported EC and executive attention were unrelated in our study was unexpected. Two studies have investigated the relationship between both constructs in TD children ( 7 - 10 years old; Simonds et al., 2007) and adolescents (16 - 17 years old; Ellis et al., 2004) using the ANT and both studies found a moderate, yet significant correlation ( $r=-.37$ and $r=-.31$, respectively). Although it is difficult to account for these inconsistent findings, a possible explanation may lie in the fact that we included clinical 
groups in our study. Perhaps, parent reports on EC and executive attention scores do not correspond as well in children with ADHD or ASD as in TD children. For example, one could argue that the presence of a diagnosis of ADHD or ASD might influence the way parents reflect on the abilities of their child to regulate their own thoughts and behavior. However, additionally analyses investigating the relationship between EC and executive attention for all groups separately, show that both constructs were unrelated in all groups, even the TD group. Although sample sizes were limited and we should be careful interpreting these results, our findings do not provide support for the above mentioned explanation. Future studies investigating the relationship between EC and executive attention in clinical groups will have to further address this issue. Overall, our findings suggest that the relationship between EC reports and indices of neural systems involved in the effortful regulation of behavior may not be as unambiguous as previously thought and that the theoretical link between both constructs may not necessarily translate in a significant relationship between EC reports and performance based executive attention scores, especially in clinical groups. Therefore, it is important to consider that executive attention scores measured by the ANT and EC reports may not be interchangeable when assessing the ability of EC in children.

Some limitations of the current study need to be acknowledged. First, the study is correlational so we cannot draw conclusions on potential causal relations between attentional networks. Secondly, all participants were boys, making it impossible to evaluate gender differences in the efficiency of attentional networks, their interrelationship, or in the relationship between executive attention and EC. Future research will have to confirm that results and conclusions can be generalized to girls. Thirdly, in our study only children with ADHD without comorbid ASD and vice versa, were included. Future research will have to show if our findings can be generalized to children diagnosed with both ADHD and ASD. Additionally, we had quite heterogeneous samples within the ADHD (i.e., children who are 
primarily of the Inattentive type as well as Hyperactive/Impulsive or combined type) and ASD groups (i.e., children with mild and severe autism). Up till now, few studies have investigated potential differences in the efficiency of attentional networks between DSM-IV subtypes of ADHD and results of those studies are inconsistent in that some studies show no differences between subtypes (e.g., Mullane et al., 2011) whereas others suggest that there may be a stronger alerting effect in ADHD of the predominantly inattentive type (ADHD IA) as compared to ADHD combined (e.g., Booth et al., 2007). Given these inconsistent findings, it would be interesting to analyze potential subgroup differences in our study. Additional analyses comparing children with ADHD IA and children with ADHD combined revealed that both subtypes of ADHD did not differ in terms of alerting, orienting, and executive attention. Additional analyses comparing two subgroups of ASD (severe versus mild ASD) indicated that there are no differences between children with mild or severe ASD in terms of the efficiency of attentional networks. However, given the small sample sizes of the ADHD and ASD subgroups, these results must be interpreted with caution. Further exploring ADHD and ASD subgroup differences in the efficiency of attentional networks might be an issue for future research. Finally, further research will have to examine whether our findings can be replicated in larger samples.

In sum, the current study demonstrated inefficient modulation of the alerting network in boys with ADHD as compared to TD peers. However, problems were limited to tonic aspects of alertness, presenting a warning signal enabled boys with ADHD to perform at a similar level as the other children, suggesting that increasing alertness may be effective in correcting the attention deficit in children with ADHD. The ASD group could not be differentiated from other groups based on performance on the modified ANT. Our findings support the idea of a functional integration of the alerting, orienting, and executive attention network and the three networks seemed to interact in a very similar way in TD children, children with ADHD, and 
children with ASD. Finally, we found no significant relationship between executive attention and EC, suggesting that the link between EC reports and indices of neural systems involved in the effortful regulation of behavior may not be as unambiguous as previously thought.

\section{References}

Adólfsdóttir, S., Sørensen, L., \& Lundervold, A. J. (2008). The attention network test: a characteristic pattern of deficits in children with ADHD. Behavioral and Brain Functions, 4, 9. doi:10.1186/1744-9081-4-9

Allen, G., \& Courchesne, E. (2001). Attention function and dysfunction in autism. Frontiers in Bioscience, 6, D105-119. doi: 10.2741/allen

American Psychiatric Association (2000). Diagnostic and statistical manual of mental disorders (DSM-IV-TR). Washington, DC: Author.

Barkley, R. A. (1998). Attention-deficit hyperactivity disorder. Scientific American, 279, 6671. doi:10.1038/scientificamerican0998-66

Berger, A., \& Posner, M. I. (2000). Pathologies of brain attentional networks. Neuroscience and Biobehavioral Reviews, 24, 3-5. doi:10.1016/S0149-7634(99)00046-9

Booth, J. E., Carlson, C. L., \& Tucker, D. M. (2007). Performance on a neurocognitive measure of alerting differentiates ADHD combined and inattentive subtypes: A preliminary report. Archives of Clinical Neuropsychology, 22, 423-432. doi:10.1016/j.acn.2007.01.017

Bush, G., Luu, P., \& Posner, M. I. (2000). Cognitive and emotional influences in the anterior cingulate cortex. Trends in Cognitive Science, 4, 215-222. doi:10.1016/S13646613(00)01483-2

Callejas, A., Lupiàñez, J., Funes, M. J., \& Tudela, P. (2005). Modulations among the alerting, orienting and executive control networks. Experimental Brain Research, 167, 27-37. doi:10.1007/s00221-005-2365-Z 
Callejas, A., Lupiãnez, J., \& Tudela, P. (2004). The three attentional networks: On their independence and interactions. Brain and Cognition, 54, 225-227. doi:10.1016/j.bandc.2004.02.012

Casagrande, M., Martella, D., Ruggiero, M. C., Maccari, L., Paloscia, C., Rosa, C., \& Pasini, A. (2012). Assessing Attentional Systems in Children with Attention Deficit Hyperactivity Disorder. Archives of Clinical Neuropsychology, 27, 30-44. doi:10.1093/arclin/acr085

Constantino, J. N. \& Gruber, C. P. (2005). Social responsiveness scale. Manual. LosAngeles, Western Psychological Services.

Courchesne, E. (1995). New evidence of cerebellar and brainstem hypoplasia in autistic infants, children and adolescents: The MR imaging study by Hashimoto and colleagues. Journal of Autism and Developmental Disorders, 25, 19-22. doi:10.1007/BF02178164

Derryberry, D., \& Reed, M. A. (2002). Anxiety-Related Attentional Biases and Their Regulation by Attentional Control. Journal of Abnormal Psychology, 111, 225-236. doi:10.1037//0021-843X.111.2.225

Ellis, L. K., \& Rothbart, M. K. (2001). Revision of the Early Adolescent Temperament Questionnaire. Poster presented at the 2001 Biennial Meeting of the Society for Research in Child Development, Minneapolis, Minnesota.

Ellis, L. K, Rothbart, M. K., \& Posner, M. I. (2004). Individual Differences in Executive Attention Predict Self-Regulation and Adolescent Psychosocial Behaviors. Annals of the New York Academy of Sciences, 1021, 337-340. doi:10.1196/annals.1308.041

Fan, J., Gu, X., Guise, K., Liu, X., Fossella, J., Wang, H. B., \& Posner, M. I. (2009). Testing the behavioral interaction and integration of attentional networks. Brain and Cognition, 70, 209-220. doi:10.1016/j.bandc.2009.02.002 
Fan, J., McCandliss, B. D., Sommer, T., Raz, A., \& Posner, M. I. (2002). Testing the efficiency and independence of attentional networks. Journal of Cognitive Neuroscience, 14, 340347. doi:10.1162/089892902317361886

Gargaro, B. A., Rinehart, N. J., Bradshaw, J. L., Tonge, B. J., \& Sheppard, D. M. (2011). Autism and ADHD: How far have we come in the comorbidity debate?, Neuroscience and Biobehavioral Review, 35, 1081-1088. doi:10.1016/j.neubiorev.2010.11.002

Gerardi-Caulton, G. (2000). Sensitivity to spatial conflict and the development of selfregulation in children 24-36 months of age. Developmental Science, 3, 397-404. doi:10.1111/1467-7687.00134

Greenwood, P. M., Fossella, J. A., \& Parasuraman, R. (2005). Specificity of the effect of a nicotinic receptor polymorphism on individual differences in visuospatial attention. Journal of Cognitive Neuroscience, 17, 1611-1620. doi:10.1162/089892905774597281

Grégoire, J. (2000). L'évaluation clinique de l'intelligence de l'enfant. Liège: Mardaga.

Haist, F., Adamo, M., Westerfield, M., Courchesne, E., \& Townsend, J. (2005). The functional neuroanatomy of spatial attention in autism spectrum disorder. Developmental Neuropsychology, 27, 425-458. doi:10.1207/s15326942dn2703_7

Halperin, J. M., \& Schulz, K. P. (2006). Revisiting the role of the prefrontal cortex in the pathophysiology of attention-deficit/hyperactivity disorder. Psychological Bulletin, 132, 560-581. doi:10.1037/0033-2909.132.4.560

Johnson, K. A., Robertson, I. H., Barry, E., Mulligan, A., Daibhis, A., Daly, M., Watchorn, A., ... Bellgrove, M. A. (2008). Impaired conflict resolution and alerting in children with ADHD: evidence from the Attention Network Task (ANT). Journal of Child Psychology and Psychiatry, 49, 1339-1347. doi:10.1111/j.1469-7610.2008.01936.x 
Keehn, B., Lincoln, A. J., Müller, R., \& Townsend, J. (2010). Attentional networks in children and adolescents with autism spectrum disorder. Journal of Child Psychology and Psychiatry, 51, 1251-1259. doi:10.1111/j.1469-7610.2010.02257.x

Konrad, K., Neufang, S., Hanisch, C., Fink, G. R., \& Herpertz-Dahlmann, B. (2006). Dysfunctional Attentional Networks in Children with Attention Deficit/Hyperactivity Disorder: Evidence from an Event-Related Functional Magnetic Resonance Imaging Study. Biological Psychiatry, 59, 643-651. doi:10.1016/j.biopsych.2005.08.013

Kort, W., Schittekatte, M., Compaan, E. L., Bosmans, M., Bleichrodt, N., Vermeir, G., Resing, W. C. M. \& Verhaeghe, P. (2002). WISC-III NL. Handleiding. Nederlandse bewerking. London: The Psychological Corporation.

Lonigan, C. J., \& Phillips, B. M. (2001). Temperamental influences on the development of anxiety disorders. In M. W. Vasey \& M. R. Dadds (Eds.), The developmental psychopathology of anxiety (pp. 60-91). New York: Oxford University Press.

Mayes, S. D., Calhoun, S. L., Mayes, R. D., \& Molitoris, S. (2012). Autism and ADHD: Overlapping and discriminating symptoms. Research in Autism Spectrum Disorders, 6, 277-285. doi:10.1016/j.rasd.2011.05.009

Mullane, J. C., Corkum, P. V., Klein, R. M., McLaughlin, E. N., \& Lawrence, M. A. (2011). Alerting, Orienting, and Executive Attention in Children With ADHD. Journal of Attention Disorders, 15, 310-320. doi:10.1177/1087054710366384

Muris, P., \& Meesters, C. (2009). Reactive and Regulative Temperament in Youths: Psychometric Evaluation of the Early Adolescent Temperament Questionnaire-Revised. Journal of Psychopathology and Behavioral Assessment, 31, 7-19. doi:10.1007/s10862008-9089-х

Murray, M. J., Mayes, S. D., \& Smith, L. A. (2011). Brief Report: Excellent Agreement Between Two Brief Autism Scales (Checklist for Autism Spectrum Disorder and Social 
Responsiveness Scale) Completed Independently by Parents and the Autism Diagnostic Interview-Revised. Journal of Autism and Developmental Disorders, 41, 1586-1590. doi:10.1007/s10803-011-1178-0

Neuhaus, A. H., Opgen-Rhein, C., Urbanek, C., Gross, M., Hahn, E., Tam Ta, T. M., Koehler, S., \& Dettling, M. (2009). Spatiotemporal mapping of sex differences during attentional processing. Human Brain Mapping, 30, 2997-3008. doi:10.1002/hbm.20724

Pelham, W. E., Gnagy, E. M., Greenslade, K. E., \& Milich, R. (1992). Teacher ratings of DSM-III-R symptoms for the disruptive behavior disorders. Journal of the American Academy of Child and Adolescent Psychiatry, 31, 210-218. doi:10.1097/00004583199203000-00006

Posner, M. I., \& Fan, J. (2004). Attention as an organ system. In J. R. Pomerantz, \& M. C. Crair (Eds.), Topics in integrative neuroscience: From cells to cognition (pp. 31-62). Cambridge: Cambridge University Press.

Posner, M. I., \& Petersen, S. E. (1990). The attention system of the human brain. Annual Review of Neuroscience, 13, 25-42. doi:10.1146/annurev.ne.13.030190.000325

Posner, M. I., \& Rothbart, M. K. (1994). Attention regulation: From mechanism to culture. In P. Bartelson, P. Elen, \& G. d'Ydewalle (Eds.), International perspectives on psychological science: Leading themes (Vol. 1, pp. 41-54). Hillsdale, NJ: Erlbaum.

Posner, M. I., \& Rothbart, M. K. (2007). Research on attention networks as a model for integration of psychological science. Annual Review of Psychology, 58, 1-23. doi: 10.1146/annurev.psych.58.110405.085516

Posner, M. I., Sheese, B. E., Odludas, Y., \& Tang, Y. Y. (2006). Analyzing and shaping human $\begin{array}{llll}\text { attentional networks. } & \text { Neural } \quad \text { Networks, } & 19, & 1422-1429 .\end{array}$ doi:10.1016/j.neunet.2006.08.004 
Rothbart, M. K., \& Bates, J. E. (2006). Temperament. In W. Damon, R. Lerner, \& N. Eisenberg (Eds.), Handbook of child psychology (6th ed.): Vol 3. Social, emotional, and personality development (pp. 99-166). New York: Wiley.

Rueda, M. R., Fan, J., McCandliss, B. D., Halparin, J. D., Gruber, D. B., Lercari, L. P., \& Posner, M. I. (2004). Development of attentional networks in childhood. Neuropsychologia, 42, 1029-1040. doi:10.1016/j.neuropsychologia.2003.12.012

Sengupta, S. M., Grizenko, N., Thakur, G. A., Bellingham, J., DeGuzman, R., Robinson, S., TerStepanian, M, ... Joober, R. (2012). Differential association between the norepinephrine transporter gene and ADHD: role of sex and subtype. Journal of Psychiatry and Neuroscience, 37, 129-137. doi:10.1503/jpn.110073

Sergeant, J., Oosterlaan, J., \& van der Meere, J. (1999). Information processing and energetic factors in attention-deficit hyperactivity. In H. C. Quay, \& A. E. Hogan (Eds.), Handbook of disruptive behavior disorders (pp. 75-104). New York: Plenum Press.

Shaffer, D., Fisher, P., Lucas, C. P., Dulcan, M. K., \& Schwab-Stone, M. E. (2000). NIMH Diagnostic Interview Schedule for Children version IV (NIMH DISC-IV): Description, differences from previous versions, and reliability of some common diagnoses. Journal of the American Academy of Child and Adolescent Psychiatry, 39, 28-38. doi:10.1097/00004583-200001000-00014

Simonds, J., Kieras, J. E., Rueda, M. R., \& Rothbart, M. K. (2007). Effortful control, executive attention, and emotional regulation in 7-10-year-old children. Cognitive Development, 22, 474-488. doi:10.1016/j.cogdev.2007.08.009

Swanson, J., Posner, M. I., Cantwell, D., Wigal, S., Crinella, F., Filipek, P.A., ... Nalcioglu, O. (2000). Attention-deficit hyperactivity disorder: symptom domain, cognitive processes and neural networks. In R. Parasuraman (Ed.), The attentive brain (pp. 445-461). Cambridge: MIT Press. 
Swettenham, J., Baron-Cohen, S., Charman, T., Cox, A., Baird, G., Drew, A., Rees, L., \& Wheelwright, S. (1998). The Frequency and Distribution of Spontaneous Attention Shifts between Social and Nonsocial Stimuli in Autistic, Typically Developing, and Nonautistic Developmentally Delayed Infants. Journal of Child Psychology and Psychiatry, 39, 747-753. doi:10.1017/S0021963098002595

Verstraeten, K., Vasey, M. W., Claes, L., \& Bijttebier, P. (2010). The Assessment of Effortful Control in Childhood: Questionnaires and the Test of Everyday Attention for Children Compared. Personality and Individual Differences, 48, 59-65. doi:10.1016/j.paid.2009.08.016 


\section{Table 1}

Efficiency scores of the attentional networks for the three groups based on mean RTs

\begin{tabular}{|c|c|c|c|c|}
\hline Network efficiency scores & $\mathrm{TD}(n=25)$ & $\operatorname{ASD}(n=25)$ & ADHD $(n=25)$ & Group differences \\
\hline Orienting & $72.68(43.84)^{\mathrm{a}}$ & $72.05(48.15)^{\mathrm{a}}$ & $71.14(47.83)^{\mathrm{a}}$ & 0.01 \\
\hline Executive attention & $121.04(47.04)^{\mathrm{a}}$ & $135.27(67.77)^{\mathrm{a}}$ & $129.00(64.24)^{\mathrm{a}}$ & 0.35 \\
\hline
\end{tabular}


(a)

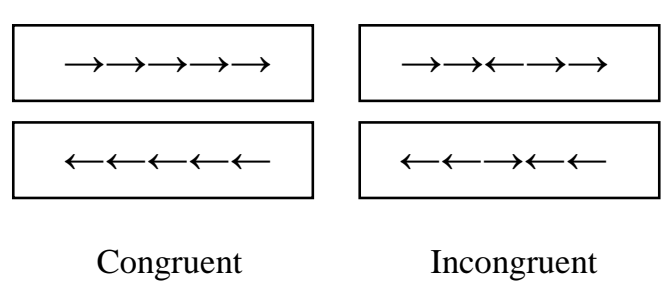

(b)

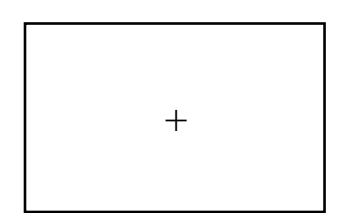

No cue

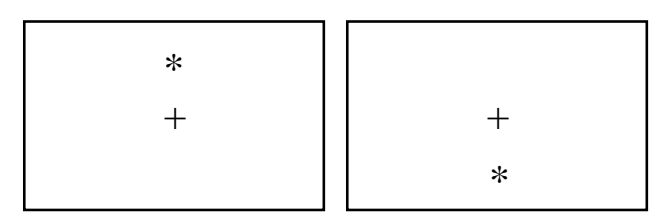

Spatial cue

(c)

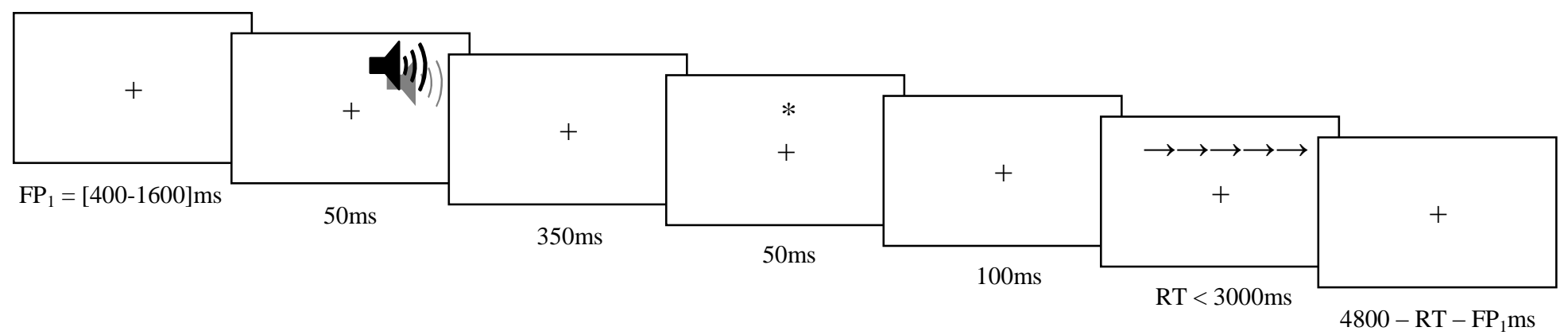

Figure 1 Illustration of the attention network test. Examples of flanker types (a), cue types (b), and the sequence and timing of a single trial (c) 
(a)

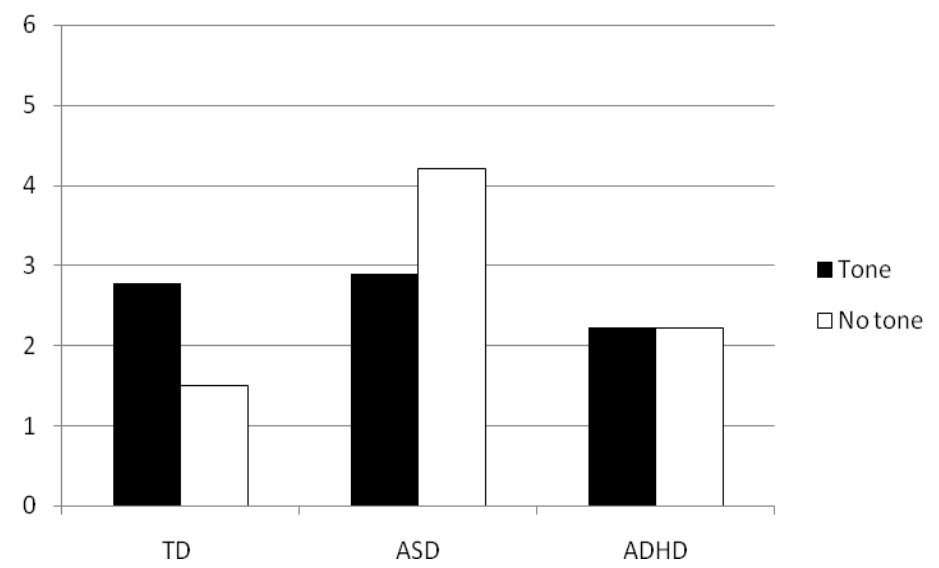

(b)

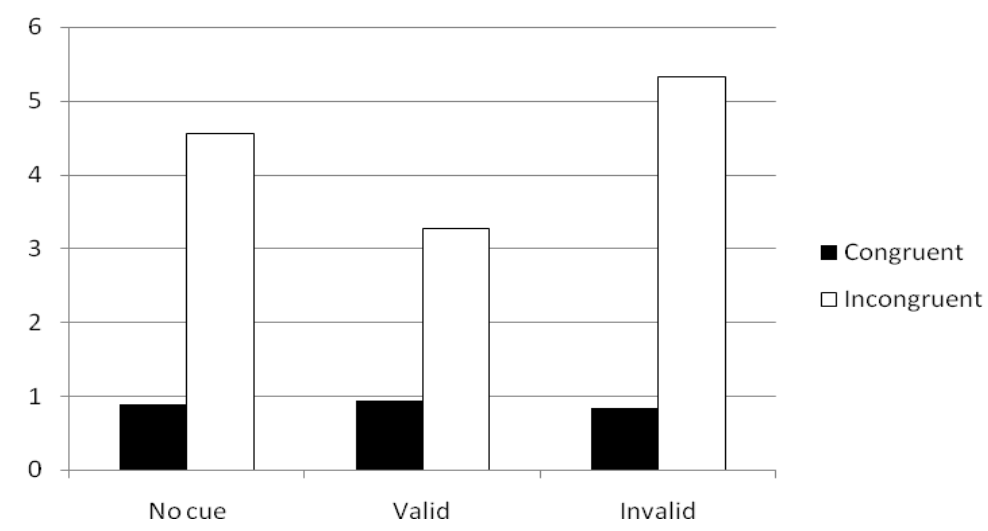

Figure 2 Bar graphs correspond to percentage incorrect responses as a function of tone and group (a), and as a function of flanker and cue (b) 
(a)

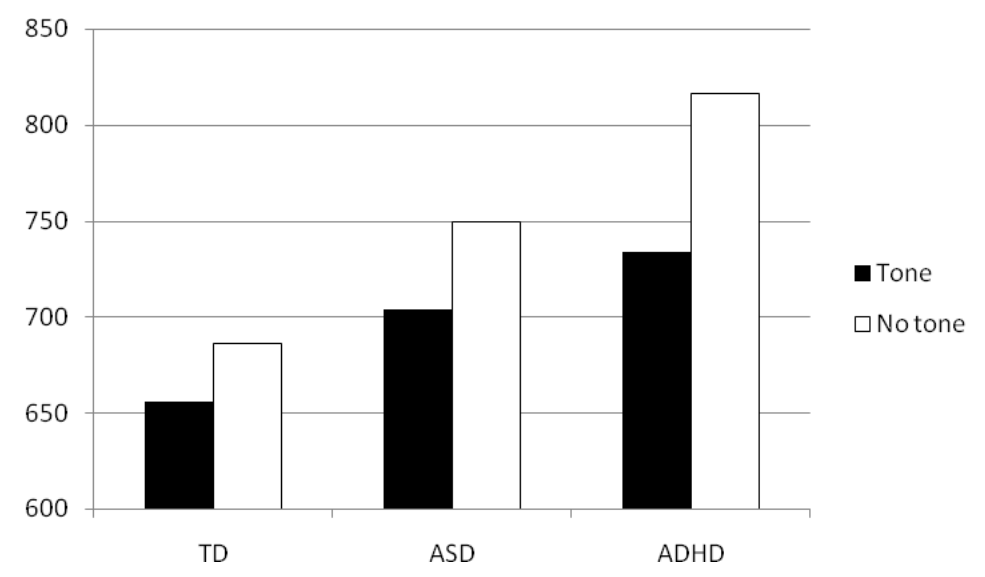

(b)

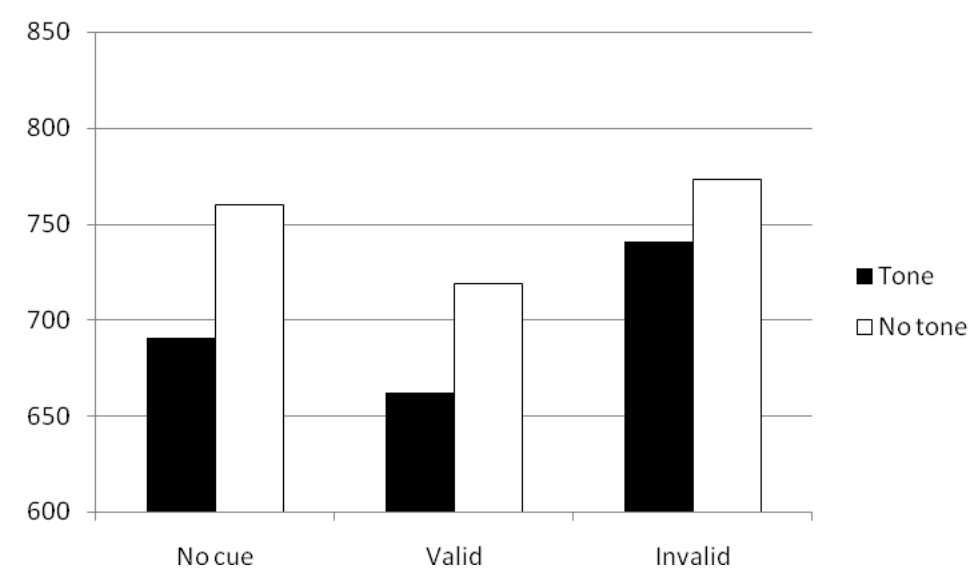

(c)

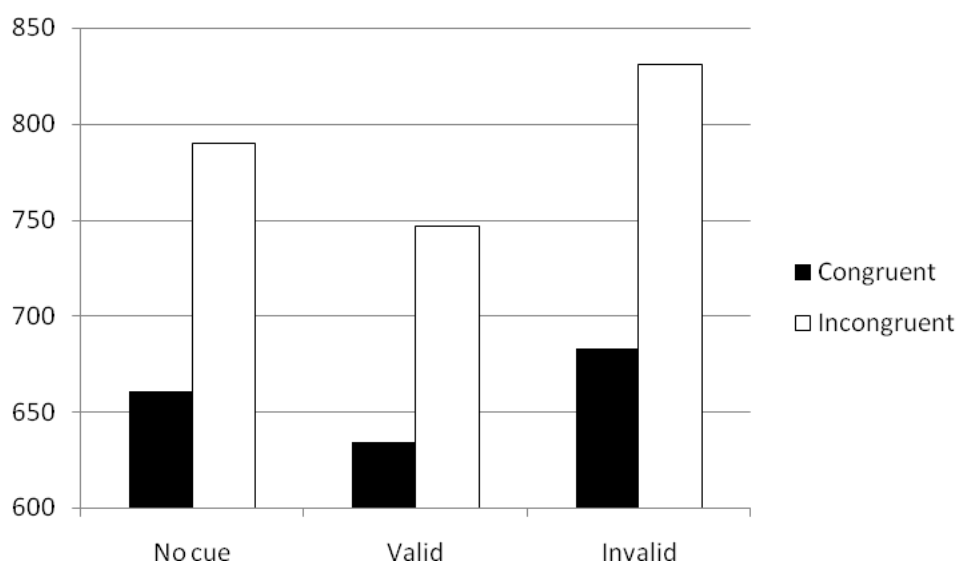

Figure 3 Bar graphs correspond to mean reaction time as a function of group and tone (a), as a function of tone and cue (b), and as a function of cue and flanker (c) 